JOURNAL OF

FUNCTION SPACES AND APPLICATIONS

Volume 3, Number 1 (2005), 91-115
(C) 2005, Scientific Horizon http://www.jfsa.net

\title{
Traces of multipliers in pairs of weighted Sobolev spaces
}

\author{
Vladimir Maz'ya and Tatyana Shaposhnikova* \\ (Communicated by Victor Burenkov)
}

2000 Mathematics Subject Classification. 46E35, 46E25.

Keywords and phrases. Multipliers, weighted Sobolev spaces, fractional Sobolev spaces.

Abstract. We prove that the pointwise multipliers acting in a pair of fractional Sobolev spaces form the space of boundary traces of multipliers in a pair of weighted Sobolev space of functions in a domain.

\section{Introduction}

By a multiplier acting from one Banach function space $S_{1}$ into another $S_{2}$ we call a function $\gamma$ such that $\gamma u \in S_{2}$ for any $u \in S_{1}$. By $M\left(S_{1} \rightarrow S_{2}\right)$ we denote the space of multipliers $\gamma: S_{1} \rightarrow S_{2}$ with the norm

$$
\|\gamma\|_{M\left(S_{1} \rightarrow S_{2}\right)}=\sup \left\{\|\gamma u\|_{S_{2}}:\|u\|_{S_{1}} \leq 1\right\} .
$$

We write $M S$ instead of $M(S \rightarrow S)$, where $S$ is a Banach function space. We shall use the same notation both for spaces of scalar and vector-valued multipliers.

*The authors were supported by grants of the Swedish National Science Foundation. 
Let $\Omega$ be a bounded domain in $\mathbb{R}^{n}$ with smooth boundary $\partial \Omega$. It is well known that the fractional Sobolev space $W_{p}^{l}(\partial \Omega)$ is the space of traces of the weighted Sobolev space $W_{p}^{s, \alpha}(\Omega)$ endowed with the norm

$$
\left(\int_{\Omega}(\operatorname{dist}(x, \partial \Omega))^{p \alpha} \sum_{\{\tau: 0 \leq|\tau| \leq s\}}\left|D^{\tau} u\right|^{p} d x\right)^{1 / p},
$$

where $\alpha=1-\{l\}-1 / p, s=[l]+1$ and $p \in(1, \infty)$ (see [5]). It is straightforward to deduce from this fact that the trace $\gamma$ of the function

$$
\Gamma \in M\left(W_{p}^{t, \beta}(\Omega) \rightarrow W_{p}^{s, \alpha}(\Omega)\right)
$$

belongs to $M\left(W_{p}^{m}(\partial \Omega) \rightarrow W_{p}^{l}(\partial \Omega)\right)$. Here $m$ and $l$ are nonintegers, $m \geq l>0, s$ and $\alpha$ are given above, $t=[m]+1, \beta=1-\{m\}-1 / p$.

In the present paper we prove that the converse assertion is also true showing that there exists an extension $\Gamma$ of $\gamma \in M\left(W_{p}^{m}(\partial \Omega) \rightarrow W_{p}^{m}(\partial \Omega)\right)$ subject to (1).

\section{The space $M\left(W_{p}^{m}\left(\mathbb{R}^{n-1}\right) \rightarrow W_{p}^{l}\left(\mathbb{R}^{n-1}\right)\right)$}

By $\mathcal{B}_{r}^{n-1}(x)$ we mean the ball $\left\{\xi \in \mathbb{R}^{n-1}:|\xi-x|<r\right\}$ and write $\mathcal{B}_{r}^{n-1}$ instead of $\mathcal{B}_{r}^{n-1}(0)$.

We need the spaces $S_{l o c}$ and $S_{\text {unif }}$ of functions on $\mathbb{R}^{n-1}$ defined as follows. By $S_{l o c}$ we denote the space

$$
\left\{u: \eta u \in S \text { for all } \eta \in C_{0}^{\infty}\left(\mathbb{R}^{n-1}\right)\right\}
$$

and by $S_{\text {unif }}$ we mean the space

$$
\left\{u: \sup _{\xi}\left\|\eta_{\xi} u\right\|_{S}<\infty\right\}
$$

where $\eta_{\xi}(x)=\eta(x-\xi), \eta \in C_{0}^{\infty}\left(\mathbb{R}^{n-1}\right), \eta=1$ on $\mathcal{B}_{1}^{n-1}$. The space $S_{\text {unif }}$ is endowed with the norm

$$
\|u\|_{S_{\text {unif }}}=\sup _{\xi}\left\|\eta_{\xi} u\right\|_{S}
$$

Let $W_{p}^{l}\left(\mathbb{R}^{n-1}\right)$ denote the fractional Sobolev space with the norm

$$
\left\|D_{p, l} u ; \mathbb{R}^{n-1}\right\|_{L_{p}}+\left\|u ; \mathbb{R}^{n-1}\right\|_{L_{p}}
$$


where

$$
\left(D_{p, l} u\right)(x)=\left(\int_{\mathbb{R}^{n-1}}\left|\nabla_{[l]} u(x+h)-\nabla_{[l]} u(x)\right|^{p}|h|^{1-n-p\{l\}} d h\right)^{1 / p},
$$

with $\nabla_{[l]}$ being the gradient of order $[l]$, i.e. $\nabla_{[l]}=\left\{\partial_{x_{1}}^{\tau_{1}}, \ldots, \partial_{x_{n-1}}^{\tau_{n-1}}\right\}$, $\tau_{1}+\ldots+\tau_{n-1}=[l]$.

In this section we collect some known properties of multipliers between fractional Sobolev spaces $W_{p}^{m}\left(\mathbb{R}^{n-1}\right)$ and $W_{p}^{l}\left(\mathbb{R}^{n-1}\right), m \geq l \geq 0$. The equivalence $a \sim b$ means that $a / b$ is bounded and separated from zero by positive constants depending on $n, p, m$, and $l$.

Proposition 1 ([3]). Let $m$ and $l$ be nonintegers, $m \geq l \geq 0$, and let $p \in(1, \infty)$.

(i) There holds

$\left\|\gamma ; \mathbb{R}^{n-1}\right\|_{M\left(W_{p}^{m} \rightarrow W_{p}^{l}\right)} \sim\left\|D_{p, l} \gamma ; \mathbb{R}^{n-1}\right\|_{M\left(W_{p}^{m} \rightarrow L_{p}\right)}+\left\|\gamma ; \mathbb{R}^{n-1}\right\|_{M\left(W_{p}^{m-l} \rightarrow L_{p}\right)}$.

(ii) If $\gamma \in M\left(W_{p}^{m}\left(\mathbb{R}^{n-1}\right) \rightarrow W_{p}^{l}\left(\mathbb{R}^{n-1}\right)\right)$ then for any multi-index $\sigma$, $|\sigma| \leq[l]$

$$
D^{\sigma} \gamma \in M\left(W_{p}^{m}\left(\mathbb{R}^{n-1}\right) \rightarrow W_{p}^{l-|\sigma|}\left(\mathbb{R}^{n-1}\right)\right)
$$

(iii) Let $0<\lambda<\mu$. Then

$$
\left\|\gamma^{\lambda / \mu} ; \mathbb{R}^{n-1}\right\|_{M\left(W_{p}^{\lambda} \rightarrow L_{p}\right)} \leq c\left\|\gamma ; \mathbb{R}^{n-1}\right\|_{M\left(W_{p}^{\mu} \rightarrow L_{p}\right)}^{\lambda / \mu} .
$$

Proposition 2 ([3]). Let $m$ and $l$ be nonintegers, $m \geq l \geq 0$, and let $p \in(1, \infty)$. Then

$$
\begin{aligned}
&\left\|\gamma ; \mathbb{R}^{n-1}\right\|_{M\left(W_{p}^{m} \rightarrow W_{p}^{l}\right)} \sim \sup _{\begin{array}{c}
e \subset \mathbb{R} n-1 \\
\text { diam(e) } \leq 1
\end{array}} \frac{\left\|D_{p, l} \gamma ; e\right\|_{L_{p}}}{\left(\operatorname{cap}_{p, m}(e)\right)^{1 / p}} \\
&+ \begin{cases}\sup _{x \in \mathbb{R}^{n-1}}\left\|\gamma ; \mathcal{B}_{1}^{n-1}(x)\right\|_{L_{1}} & \text { for } m>l, \\
\left\|\gamma ; \mathbb{R}^{n-1}\right\|_{L_{\infty}} & \text { for } m=l,\end{cases}
\end{aligned}
$$

where $e$ is a compact set in $\mathbb{R}^{n-1}$ and $\operatorname{cap}_{p, m}(e)$ is the $(p, m)$-capacity of $e$ defined by

$$
\operatorname{cap}_{p, m}(e)=\inf \left\{\left\|u ; \mathbb{R}^{n-1}\right\|_{W_{p}^{m}}^{p}: u \in C_{0}^{\infty}\left(\mathbb{R}^{n-1}\right), u \geq 1 \text { on } e\right\}
$$

For $l=0$ one should replace $D_{p, l} \gamma$ by $\gamma$. 
Upper estimates for the norm in $M\left(W_{p}^{m}\left(\mathbb{R}^{n-1}\right) \rightarrow W_{p}^{l}\left(\mathbb{R}^{n-1}\right)\right)$ are given in the following assertion. By mes ${ }_{n-1}$ we mean the $(n-1)$-dimensional Lebesgue measure of a compact set $e$.

Proposition 3 ([3]). Let $m$ and $l$ be nonintegers, $m \geq l \geq 0$, and let $p \in(1, \infty)$.

(i) If $m p<n-1$, then

$$
\begin{aligned}
c\left\|\gamma ; \mathbb{R}^{n-1}\right\|_{M\left(W_{p}^{m} \rightarrow W_{p}^{l}\right)} \leq & \sup _{\substack{e \in \mathbb{R} n \\
\operatorname{diam}(e) \leq 1}} \frac{\left\|D_{p, l} \gamma ; e\right\|_{L_{p}}}{\left(\operatorname{mes}_{n-1}(e)\right)^{1 / p-m /(n-1)}} \\
& + \begin{cases}\sup _{x \in \mathbb{R}^{n-1}}\left\|\gamma ; \mathcal{B}_{1}^{n-1}(x)\right\|_{L_{1}} & \text { for } m>l, \\
\left\|\gamma ; \mathbb{R}^{n-1}\right\|_{L_{\infty}} & \text { for } m=l .\end{cases}
\end{aligned}
$$

(ii) If $m p=n-1$, then

$$
\begin{array}{r}
c\left\|\gamma ; \mathbb{R}^{n-1}\right\|_{M\left(W_{p}^{m} \rightarrow W_{p}^{l}\right) \leq} \sup _{\substack{e \subset \mathbb{R} n-1 \\
\operatorname{diam}(e) \leq 1}}\left(\log \frac{2^{n-1}}{\operatorname{mes}_{n-1}(e)}\right)^{1-1 / p}\left\|D_{p, l} \gamma ; e\right\|_{L_{p}} \\
+ \begin{cases}\sup _{x \in \mathbb{R}^{n-1}}\left\|\gamma ; \mathcal{B}_{1}^{n-1}(x)\right\|_{L_{1}} & \text { for } m>l, \\
\left\|\gamma ; \mathbb{R}^{n-1}\right\|_{L_{\infty}} & \text { for } m=l .\end{cases} \\
\end{array}
$$

Now we list lower estimates for the norm in $M\left(W_{p}^{m} \rightarrow W_{p}^{l}\right)$.

Proposition 4. [3] Let $m$ and $l$ be nonintegers, $m \geq l \geq 0$, and let $p \in(1, \infty)$.

(i) If $m p<n-1$, then

$$
\begin{aligned}
c\left\|\gamma ; \mathbb{R}^{n-1}\right\|_{M\left(W_{p}^{m} \rightarrow W_{p}^{l}\right)} \geq & \sup _{\substack{x \in \mathbb{R}^{n-1} \\
r \in(0,1)}} \frac{\left\|D_{p, l} \gamma ; \mathcal{B}_{r}^{n-1}(x)\right\|_{L_{p}}}{r^{(n-1) / p-m}} \\
& + \begin{cases}\sup _{x \in \mathbb{R}^{n-1}}\left\|\gamma ; \mathcal{B}_{1}^{n-1}(x)\right\|_{L_{1}} & \text { for } m>l, \\
\left\|\gamma ; \mathbb{R}^{n-1}\right\|_{L_{\infty}} & \text { for } m=l .\end{cases}
\end{aligned}
$$

(ii) If $m p=n-1$, then

$$
\begin{aligned}
c\left\|\gamma ; \mathbb{R}^{n-1}\right\|_{M\left(W_{p}^{m} \rightarrow W_{p}^{l}\right)} \geq \sup _{\substack{x \in \mathbb{R}_{n} \\
r \in(0,1)}}\left(\log \frac{2}{r}\right)^{1-1 / p}\left\|D_{p, l} \gamma ; \mathcal{B}_{r}^{n-1}(x)\right\|_{L_{p}} \\
+ \begin{cases}\sup _{x \in \mathbb{R}^{n-1}}\left\|\gamma ; \mathcal{B}_{1}^{n-1}(x)\right\|_{L_{1}} & \text { for } m>l, \\
\left\|\gamma ; \mathbb{R}^{n-1}\right\|_{L_{\infty}} & \text { for } m=l .\end{cases}
\end{aligned}
$$




\section{Multipliers in pairs of weighted Sobolev spaces in $\mathbb{R}_{+}^{n}$}

3.1 Preliminary facts. Let $\mathbb{R}_{+}^{n}$ denote the upper half-space $\{z=(x, y)$ : $\left.x \in \mathbb{R}^{n-1}, y>0\right\}$. We introduce the weighted Sobolev space $W_{p}^{s, \alpha}\left(\mathbb{R}_{+}^{n}\right)$ with the norm

$$
\left\|(\min \{1, y\})^{\alpha} \nabla_{s} U ; \mathbb{R}_{+}^{n}\right\|_{L_{p}}+\left\|(\min \{1, y\})^{\alpha} U ; \mathbb{R}_{+}^{n}\right\|_{L_{p}},
$$

where $s$ is nonnegative integer. We always asume that $-1<\alpha p<p-1$.

It is well known that the fractional Sobolev space $W_{p}^{l}\left(\mathbb{R}^{n-1}\right)$, is the space of traces on $\mathbb{R}^{n-1}$ of functions in the space $W_{p}^{s, \alpha}\left(\mathbb{R}_{+}^{n}\right)$, where $s=[l]+1$, $\alpha=1-\{l\}-1 / p$, and $p \in(1, \infty)$ (see [5]). We show that a similar result holds for spaces of pointwise multipliers acting in a pair of fractional Sobolev spaces. To be precise, we prove that for all noninteger $m$ and $l$, $m \geq l>0$, the multiplier space $M\left(W_{p}^{m}\left(\mathbb{R}^{n-1}\right) \rightarrow W_{p}^{l}\left(\mathbb{R}^{n-1}\right)\right)$ is the space of traces on $\mathbb{R}^{n-1}$ of functions in $M\left(W_{p}^{t, \beta}\left(\mathbb{R}_{+}^{n}\right) \rightarrow W_{p}^{s, \alpha}\left(\mathbb{R}_{+}^{n}\right)\right)$, where $s$ and $\alpha$ are as above and $\beta=1-\{m\}-1 / p, t=[m]+1$. Different positive constants depending on $n, p, l, m, s, t$ will be denoted by $c$. We shall omit $\mathbb{R}_{+}^{n}$ in notations of norms.

We introduce the notion of $(p, s, \alpha)$-capacity of a compact set $e \subset \mathbb{R}_{+}^{n}$ :

$$
\operatorname{cap}_{p, s, \alpha}(e)=\inf \left\{\left\|U ; \mathbb{R}_{+}^{n}\right\|_{W_{p}^{s, \alpha}}^{p}: U \in C_{0}^{\infty}\left(\mathbb{R}_{+}^{n}\right), \quad U \geq 1 \text { on } e\right\} .
$$

The following result is essentially known (see [2], Sections 8.1, 8.2).

Proposition 5. Let $k$ be a nonnegative integer, $-1<\beta p<p-1$, and let $1<p<\infty$. Then $\Gamma \in M\left(W_{p}^{k, \beta}\left(\mathbb{R}_{+}^{n}\right) \rightarrow W_{p}^{0, \alpha}\left(\mathbb{R}_{+}^{n}\right)\right)$ if and only if

$$
\sup _{\substack{e \in \mathbb{R}_{+}^{n} \\ \operatorname{diam}(e) \leq 1}} \frac{\left\|(\min \{1, y\})^{\alpha} \Gamma ; e\right\|_{L_{p}}}{\left(\operatorname{cap}_{p, k, \beta}(e)\right)^{1 / p}}<\infty .
$$

The equivalence relation

$$
\|\Gamma\|_{M\left(W_{p}^{k, \beta} \rightarrow W_{p}^{0, \alpha}\right)} \sim \sup _{\substack{e \subset \mathbb{R}^{n} \\ \operatorname{diam}(e) \leq 1}} \frac{\left\|(\min \{1, y\})^{\alpha} \Gamma ; e\right\|_{L_{p}}}{\left(\operatorname{cap}_{p, k, \beta}(e)\right)^{1 / p}}
$$

is valid.

We shall use some general properties of multipliers. We start with the inequality

$$
\|\Gamma\|_{M\left(W_{p}^{t-j, \beta} \rightarrow W_{p}^{s-j, \alpha}\right)} \leq c\|\Gamma\|_{M\left(W_{p}^{t, \beta} \rightarrow W_{p}^{s, \alpha}\right)}^{(s-j) / s}\|\Gamma\|_{M\left(W_{p}^{t-s, \beta} \rightarrow W_{p}^{0, \alpha}\right)}^{j / s},
$$


where $0 \leq j \leq s,-1<\alpha p<p-1,-1<\beta p<p-1$, which follows from the interpolation property of weighted Sobolev spaces (see [4], Section 3.4.2).

The next assertion contains inequalities between multipliers and their mollifiers in variables $x$.

Lemma 1. Let $\Gamma_{\rho}$ denote a mollifier of a function $\Gamma$ defined by

$$
\Gamma_{\rho}(x, y)=\rho^{-n+1} \int_{\mathbb{R}^{n-1}} K\left(\rho^{-1}(x-\xi)\right) \Gamma(\xi, y) d \xi,
$$

where $K \in C_{0}^{\infty}\left(\mathcal{B}_{1}^{n-1}\right), K \geq 0$, and $\left\|K ; \mathbb{R}^{n-1}\right\|_{L_{1}}=1$. Then

$$
\left\|\Gamma_{\rho}\right\|_{M\left(W_{p}^{t, \beta} \rightarrow W_{p}^{s, \alpha}\right)} \leq\|\Gamma\|_{M\left(W_{p}^{t, \beta} \rightarrow W_{p}^{s, \alpha}\right)} \leq \liminf _{\rho \rightarrow 0}\left\|\Gamma_{\rho}\right\|_{M\left(W_{p}^{t, \beta} \rightarrow W_{p}^{s, \alpha}\right)}
$$

Proof. Let $U \in C_{0}^{\infty}$. By Minkowski's inequality

$$
\begin{aligned}
& \left(\int_{\mathbb{R}_{+}^{n}}(\min \{1, y\})^{p \alpha}\left|\nabla_{j, z} \int_{\mathbb{R}^{n-1}} \rho^{-n} K(\xi / \rho) \Gamma(x-\xi, y) U(x, y) d \xi\right|^{p} d z\right)^{1 / p} \\
& \leq \int_{\mathbb{R}^{n-1}} \rho^{-n} K(\xi / \rho)\left(\int_{\mathbb{R}_{+}^{n}}(\min \{1, y\})^{p \alpha}\left|\nabla_{j, z}(\Gamma(x, y) U(x+\xi, y))\right|^{p} d z\right)^{1 / p} d \xi
\end{aligned}
$$

where $j=0, s$. Therefore,

$$
\begin{aligned}
& \left\|\Gamma_{\rho} u\right\|_{W_{p}^{s, \alpha}} \leq\|\Gamma\|_{M\left(W_{p}^{t, \beta} \rightarrow W_{p}^{s, \alpha}\right)} \\
& \times \int_{\mathbb{R}^{n-1}} \rho^{-n} K(\xi / \rho)\left\{\left(\int_{\mathbb{R}_{+}^{n}}(\min \{1, y\})^{p \beta}\left|\nabla_{t, z} U(x+\xi, y)\right|^{p} d z\right)^{1 / p}\right. \\
& \left.+\left(\int_{\mathbb{R}_{+}^{n}}(\min \{1, y\})^{p \beta}|U(x+\xi, y)|^{p} d z\right)^{1 / p}\right\} d \xi \\
& \leq\|\Gamma\|_{M\left(W_{p}^{t, \beta} \rightarrow W_{p}^{s, \alpha}\right)}\|U\|_{W_{p}^{t, \beta}} .
\end{aligned}
$$

This gives the left inequality (6). The right inequality (6) follows from

$$
\|\Gamma u\|_{W_{p}^{s, \alpha}}=\liminf _{\rho \rightarrow 0}\left\|\Gamma_{\rho} U\right\|_{W_{p}^{s, \alpha}} \leq \liminf _{\rho \rightarrow 0}\left\|\Gamma_{\rho}\right\|_{M\left(W_{p}^{t, \beta} \rightarrow W_{p}^{s, \alpha}\right)}\|U\|_{W_{p}^{t, \beta}} .
$$

The proof is complete.

Lemma 2. Let $\Gamma \in L_{p, l o c}, p \in(1, \infty),-1<\beta p<p-1$, and let $U$ be an arbitrary function in $C_{0}^{\infty}\left(\mathbb{R}_{+}^{n}\right)$. The best constant in the inequality

$$
\left\|(\min \{1, y\})^{\alpha} \Gamma \nabla_{s} U\right\|_{L_{p}}+\left\|(\min \{1, y\})^{\alpha} \Gamma U\right\|_{L_{p}} \leq C\|U\|_{W_{p}^{t, \beta}}
$$


is equivalent to the norm $\|\Gamma\|_{M\left(W_{p}^{t-s, \beta} \rightarrow W_{p}^{0, \alpha}\right)}$.

Proof. The estimate $C \leq c\|\Gamma\|_{M\left(W_{p}^{t-s, \beta} \rightarrow W_{p}^{0, \alpha}\right)}$ is obvious. To derive the converse estimate, we introduce a function $x \rightarrow \sigma$ which is positive on $[0, \infty)$ and is equal to $x$ for $x>1$. For any $U \in C_{0}^{\infty}\left(\mathbb{R}_{+}^{n}\right)$ there holds

$$
U=(-\Delta)^{s}(\sigma(-\Delta))^{-[l]-1} u+T(-\Delta) u,
$$

where $T$ is a function in $C_{0}^{\infty}([0, \infty))$. Since

$$
(-\Delta)^{s}=(-1)^{s} \sum_{|\tau|=s} \frac{s !}{\tau !} D^{2 \tau},
$$

it follows from (7) and the theorem on the boundedness of convolution operators in weighted $L_{p}$ spaces (see [1]) that

$$
\begin{gathered}
\int_{\mathbb{R}_{+}^{n}}(\min \{1, y\})^{p \alpha}|\Gamma(z) U(z)|^{p} d z \\
\leq c C\left(\left\|\nabla_{s}(\sigma(-\Delta))^{-s} U\right\|_{W_{p}^{t, \beta}}^{p}+\|T U\|_{W_{p}^{t, \beta}}^{p} \leq c C\|U\|_{W_{p}^{t-s, \beta}}^{p} .\right.
\end{gathered}
$$

The proof is complete.

\section{Characterisation of the space $M\left(W_{p}^{t, \beta} \rightarrow W_{p}^{s, \alpha}\right)$}

Here we derive necessary and sufficient conditions for a function to belong to the space $M\left(W_{p}^{t, \beta} \rightarrow W_{p}^{s, \alpha}\right)$ for $p \in(1, \infty)$ with $\alpha$ and $\beta$ satisfying

$$
-1<\alpha p<p-1, \quad-1<\beta p<p-1, \quad t \geq s .
$$

These inequalities will be assumed everywhere. We start with an assertion on derivatives of multipliers. We shall omit $\mathbb{R}_{+}^{n+1}$ in notations of spaces, norms, and integrals.

Lemma 3. Suppose

$$
\Gamma \in M\left(W_{p}^{t, \beta} \rightarrow W_{p}^{s, \alpha}\right) \cap M\left(W_{p}^{t-s, \beta} \rightarrow W_{p}^{0, \alpha}\right), \quad p \in(1, \infty) .
$$

Then $D^{\sigma} \Gamma \in M\left(W_{p}^{t, \beta} \rightarrow W_{p}^{s-|\sigma|, \alpha}\right)$ for any multiindex $\sigma$ of order $|\sigma| \leq s$ and

$\left\|D^{\sigma} \Gamma\right\|_{M\left(W_{p}^{t, \beta} \rightarrow W_{p}^{s-|\sigma|, \alpha}\right)}$

$$
\leq \varepsilon\|\Gamma\|_{M\left(W_{p}^{t-s, \beta} \rightarrow W_{p}^{0, \alpha}\right)}+c(\varepsilon)\|\Gamma\|_{M\left(W_{p}^{t, \beta} \rightarrow W_{p}^{s, \alpha}\right)},
$$

where $\varepsilon$ is an arbitrary positive number. 
Proof. Let $U \in W_{p}^{s, \alpha}$ and let $\varphi$ be an arbitrary function in $C_{0}^{\infty}$. Applying Leibniz formula

$$
D^{\sigma}(\varphi U)=\sum_{\{\tau: \sigma \geq \tau \geq 0\}} \frac{\sigma !}{\tau !(\sigma-\tau) !} D^{\tau} \varphi D^{\sigma-\tau} U
$$

we find

$$
\begin{aligned}
\int \varphi U(-D)^{\sigma} \Gamma d z & =\int \Gamma D^{\sigma}(\varphi U) d z \\
& =\sum_{\{\tau: \sigma \geq \tau \geq 0\}} \frac{\sigma !}{\tau !(\sigma-\tau) !} \Gamma D^{\tau} \varphi D^{\sigma-\tau} U d z \\
& =\int \varphi \sum_{\{\beta: \sigma \geq \tau \geq 0\}} \frac{\sigma !}{\tau !(\sigma-\tau) !}(-D)^{\tau}\left(\Gamma D^{\sigma-\tau} U\right) d z
\end{aligned}
$$

Therefore,

$$
U D^{\sigma} \Gamma=\sum_{\{\tau: \sigma \geq \tau \geq 0\}} \frac{\sigma !}{\tau !(\sigma-\tau) !}(D)^{\tau}\left(\Gamma(-D)^{\sigma-\tau} U\right),
$$

which implies the estimate

$$
\left\|U D^{\sigma} \Gamma\right\|_{W_{p}^{s-|\sigma|, \alpha}} \leq c \sum_{\{\tau: \sigma \geq \tau \geq 0\}}\left\|\Gamma D^{\sigma-\tau} U\right\|_{W_{p}^{s-|\sigma|+|\tau|, \alpha}} .
$$

Hence, it suffices to prove (9) for $|\sigma|=1$. We have

$$
\begin{aligned}
\|U \nabla \Gamma\|_{W_{p}^{s-1, \alpha}} & \leq\|U \Gamma\|_{W_{p}^{s, \alpha}}+\|\Gamma \nabla U\|_{W_{p}^{s-1, \alpha}} \\
& \leq\left(\|\Gamma\|_{M\left(W_{p}^{t, \beta} \rightarrow W_{p}^{s, \alpha}\right)}+\|\Gamma\|_{M\left(W_{p}^{t-1, \beta} \rightarrow W_{p}^{s-1, \alpha}\right)}\right)\|U\|_{W_{p}^{t, \beta} .}
\end{aligned}
$$

Estimating the norm $\|\Gamma\|_{M\left(W_{p}^{t-1, \beta} \rightarrow W_{p}^{s-1, \alpha}\right)}$ by (5) we arrive at (9).

We pass now to two-sided estimates of norms in $M\left(W_{p}^{t, \beta} \rightarrow W_{p}^{s, \alpha}\right)$, $p \in(1, \infty)$, given in terms of the spaces $M\left(W_{p}^{k, \beta} \rightarrow W_{p}^{0, \alpha}\right)$. We start with lower estimates.

Lemma 4. Let $\Gamma \in M\left(W_{p}^{t, \beta} \rightarrow W_{p}^{s, \alpha}\right)$. Then

$$
\left\|\nabla_{s} \Gamma\right\|_{M\left(W_{p}^{t, \beta} \rightarrow W_{p}^{0, \alpha}\right)}+\|\Gamma\|_{M\left(W_{p}^{t-s, \beta} \rightarrow W_{p}^{0, \alpha}\right)} \leq c\|\Gamma\|_{M\left(W_{p}^{t, \beta} \rightarrow W_{p}^{s, \alpha}\right)} .
$$

Proof. Suppose first that $\Gamma \in M\left(W_{p}^{t-s, \beta} \rightarrow W_{p}^{0, \alpha}\right)$. We have

$$
\left\|\Gamma \nabla_{s} U\right\|_{W_{p}^{0, \alpha}} \leq\|\Gamma\|_{M\left(W_{p}^{t, \beta} \rightarrow W_{p}^{s, \alpha}\right)}\|U\|_{W_{p}^{t, \beta}}+c \sum_{\substack{|\sigma|+|\tau|=s, \tau \neq 0}}\left\|D^{\sigma} U D^{\tau} \Gamma\right\|_{W_{p}^{0, \alpha}}
$$


(11)

$$
\leq\left(\|\Gamma\|_{M\left(W_{p}^{t, \beta} \rightarrow W_{p}^{s, \alpha}\right)}+c \sum_{j=1}^{s}\left\|\nabla_{j} \Gamma\right\|_{M\left(W_{p}^{t-s+j} \rightarrow W_{p}^{0, \alpha}\right)}\right)\|U\|_{W_{p}^{t, \beta} .} .
$$

By Lemma 3,

$$
\begin{aligned}
& \left\|\nabla_{j} \Gamma\right\|_{M\left(W_{p}^{t-s+j, \beta} \rightarrow W_{p}^{0, \alpha}\right)} \\
& \quad \leq \varepsilon\|\Gamma\|_{M\left(W_{p}^{t-s, \beta} \rightarrow W_{p}^{0, \alpha}\right)}+c(\varepsilon)\|\Gamma\|_{M\left(W_{p}^{t-s+j, \beta} \rightarrow W_{p}^{j, \alpha}\right)} .
\end{aligned}
$$

Estimating the last norm by (5) we obtain

$$
\begin{aligned}
& \left\|\nabla_{j} \Gamma\right\|_{M\left(W_{p}^{t-s+j, \beta} \rightarrow W_{p}^{0, \alpha}\right)} \\
& \quad \leq \varepsilon\|\Gamma\|_{M\left(W_{p}^{t-s, \beta} \rightarrow W_{p}^{0, \alpha}\right)}+c(\varepsilon)\|\Gamma\|_{M\left(W_{p}^{t, \beta} \rightarrow W_{p}^{s, \alpha}\right)} .
\end{aligned}
$$

Substitution of this into (11) gives

$$
\begin{aligned}
& \left\|\Gamma \nabla_{s} U\right\|_{W_{p}^{0, \alpha}} \\
& \quad \leq\left(\varepsilon\|\Gamma\|_{M\left(W_{p}^{t-s, \beta} \rightarrow W_{p}^{0, \alpha}\right)}+c(\varepsilon)\|\Gamma\|_{M\left(W_{p}^{t, \beta} \rightarrow W_{p}^{s, \alpha}\right)}\right)\|U\|_{W_{p}^{t, \beta} .}
\end{aligned}
$$

Besides,

$$
\|\Gamma U\|_{W_{p}^{0, \alpha}} \leq\|\Gamma\|_{M\left(W_{p}^{t, \beta} \rightarrow W_{p}^{s, \alpha}\right)}\|U\|_{W_{p}^{t, \beta}} .
$$

Summing up two last estimates and applying Lemma 2 we arrive at

$$
\|\Gamma\|_{M\left(W_{p}^{t-s, \beta} \rightarrow W_{p}^{0, \alpha}\right)} \leq \varepsilon\|\Gamma\|_{M\left(W_{p}^{t-s, \beta} \rightarrow W_{p}^{0, \alpha}\right)}+c(\varepsilon)\|\Gamma\|_{M\left(W_{p}^{t, \beta} \rightarrow W_{p}^{s, \alpha}\right)} .
$$

Hence,

$$
\|\Gamma\|_{M\left(W_{p}^{t-s, \beta} \rightarrow W_{p}^{0, \alpha}\right)} \leq c\|\Gamma\|_{M\left(W_{p}^{t, \beta} \rightarrow W_{p}^{s, \alpha}\right)} .
$$

Now, we are going to remove the assumption $\Gamma \in M\left(W_{p}^{t-s, \beta} \rightarrow W_{p}^{0, \alpha}\right)$. Since $\Gamma \in M\left(W_{p}^{t, \beta} \rightarrow W_{p}^{s, \alpha}\right)$, then

$$
\|\Gamma \eta\|_{W_{p}^{s, \alpha}} \leq c\|\eta\|_{W_{p}^{t, \beta}},
$$

where $\eta \in C_{0}^{\infty}\left(\mathcal{B}_{2}^{n}(z)\right), \eta=1$ on $\mathcal{B}_{1}^{n}(z)$, and $z$ is an arbitrary point in $\mathbb{R}_{+}^{n}$. Hence $\Gamma \in W_{p, \text { unif }}^{s, \alpha}\left(\mathbb{R}_{+}^{n}\right)$ which implies that for any $(n-1)$-dimensional multiindex $\tau$ the derivative $D_{x}^{\tau} \Gamma_{\rho}$ belongs to $W_{p, \text { unif }}^{s, \alpha}\left(\mathbb{R}_{+}^{n}\right)$. Therefore, $\Gamma_{\rho} \in L_{\infty}\left(\mathbb{R}_{+}^{n}\right)$ which in its turn guarantees that $\Gamma_{\rho} \in M\left(W_{p}^{t-s, \beta} \rightarrow W_{p}^{0, \alpha}\right)$. 
Thus, we may put $\Gamma_{\rho}$ into (15) in order to obtain

$$
\left\|\Gamma_{\rho}\right\|_{M\left(W_{p}^{t-s, \beta} \rightarrow W_{p}^{0, \alpha}\right)} \leq c\left\|\Gamma_{\rho}\right\|_{M\left(W_{p}^{t, \beta} \rightarrow W_{p}^{s, \alpha}\right)} .
$$

Letting $\rho \rightarrow 0$ and using Lemma 1 we arrive at (15) for all $\Gamma \in M\left(W_{p}^{t, \beta} \rightarrow\right.$ $\left.W_{p}^{s, \alpha}\right)$.

To estimate the first term in the right-hand side of (10), we combine (15) with (12) for $j=s$.

The estimate converse to (10) is contained in the following lemma.

Lemma 5. Let $\Gamma \in M\left(W_{p}^{t-s, \beta} \rightarrow W_{p}^{0, \alpha}\right)$ and let $\nabla_{s} \Gamma \in M\left(W_{p}^{t, \beta} \rightarrow\right.$ $\left.W_{p}^{0, \alpha}\right)$. Then $\Gamma \in M\left(W_{p}^{t, \beta} \rightarrow W_{p}^{s, \alpha}\right)$ and the estimate

(16) $\|\Gamma\|_{M\left(W_{p}^{t, \beta} \rightarrow W_{p}^{s, \alpha}\right)} \leq c\left(\left\|\nabla_{s} \Gamma\right\|_{M\left(W_{p}^{t, \beta} \rightarrow W_{p}^{0, \alpha}\right)}+\|\Gamma\|_{M\left(W_{p}^{t-s, \beta} \rightarrow W_{p}^{0, \alpha}\right)}\right)$

is valid.

Proof. By Lemma 4 and (5) we have

$$
\left\|\nabla_{j} \Gamma\right\|_{M\left(W_{p}^{t-s+j, \beta} \rightarrow W_{p}^{0, \alpha}\right)} \leq c\|\Gamma\|_{M\left(W_{p}^{t-s+j, \beta} \rightarrow W_{p}^{j, \alpha}\right)}
$$

$$
\leq c\|\Gamma\|_{M\left(W_{p}^{t, \beta} \rightarrow W_{p}^{s, \alpha}\right)}^{j / s}\|\Gamma\|_{M\left(W_{p}^{t-s, \beta} \rightarrow W_{p}^{0, \alpha}\right)}^{1-j / s},
$$

where $j=1, \ldots, s$. For any $U \in C_{0}^{\infty}$,

$$
\begin{aligned}
\left\|(\min \{1, y\})^{\alpha} \nabla_{s}(\Gamma U)\right\|_{L_{p}} \leq & c \sum_{j=0}^{s}\left\|(\min \{1, y\})^{\alpha}\left|\nabla_{j} \Gamma\right|\left|\nabla_{s-j} U\right|\right\|_{L_{p}} \\
\leq & c\left(\left\|\nabla_{s} \Gamma\right\|_{M\left(W_{p}^{t, \beta} \rightarrow W_{p}^{0, \alpha}\right)}+\|\Gamma\|_{M\left(W_{p}^{t-s, \beta} \rightarrow W_{p}^{0, \alpha}\right)}\right. \\
& \left.+\sum_{j=1}^{s-1}\left\|\nabla_{j} \Gamma\right\|_{M\left(W_{p}^{t-s+j, \beta} \rightarrow W_{p}^{0, \alpha}\right)}\right)\|U\|_{W_{p}^{t, \beta} .}
\end{aligned}
$$

This and (17) imply

$$
\begin{aligned}
& \left\|(\min \{1, y\})^{\alpha} \nabla_{s}(\Gamma U)\right\|_{L_{p}} \\
& \quad \leq c\left(\left\|\nabla_{s} \Gamma\right\|_{M\left(W_{p}^{t, \beta} \rightarrow W_{p}^{0, \alpha}\right)}+\|\Gamma\|_{M\left(W_{p}^{t-s, \beta} \rightarrow W_{p}^{0, \alpha}\right)}\right)\|U\|_{W_{p}^{t, \beta}} .
\end{aligned}
$$

It remains to note that

$$
\left\|(\min \{1, y\})^{\alpha} \Gamma U\right\|_{L_{p}} \leq\|\Gamma\|_{M\left(W_{p}^{t-s, \beta} \rightarrow W_{p}^{0, \alpha}\right)}\|U\|_{W_{p}^{t-s, \beta}}
$$


The proof is complete.

Using Lemmas 4 and 5 we arrive at the following description of the space $M\left(W_{p}^{t, \beta}\left(\mathbb{R}_{+}^{n}\right) \rightarrow W_{p}^{s, \alpha}\left(\mathbb{R}_{+}^{n}\right)\right)$.

Theorem 1. A function $\Gamma$ belongs to the space $M\left(W_{p}^{t, \beta} \rightarrow W_{p}^{s, \alpha}\right)$ if and only if $\Gamma \in W_{p, \text { loc }}^{s, \alpha}, \Gamma \in M\left(W_{p}^{t-s, \beta} \rightarrow W_{p}^{0, \alpha}\right)$, and $\nabla_{s} \Gamma \in M\left(W_{p}^{t, \beta} \rightarrow W_{p}^{0, \alpha}\right)$. Moreover,

$$
\|\Gamma\|_{M\left(W_{p}^{t, \beta} \rightarrow W_{p}^{s, \alpha}\right)} \sim\left\|\nabla_{s} \Gamma\right\|_{M\left(W_{p}^{t, \beta} \rightarrow W_{p}^{0, \alpha}\right)}+\|\Gamma\|_{M\left(W_{p}^{t-s, \beta} \rightarrow W_{p}^{0, \alpha}\right)} .
$$

The equivalence relation (4) enables one to reformulate Theorem 1.

Theorem 2. A function $\Gamma$ belongs to the space $M\left(W_{p}^{t, \beta} \rightarrow W_{p}^{s, \alpha}\right)$ if and only if $\Gamma \in W_{p, \text { loc }}^{s, \alpha}$ and for any compact set $e \subset \mathbb{R}_{+}^{n}$

Moreover,

$$
\begin{aligned}
& \left\|(\min \{1, y\})^{\alpha} \nabla_{s} \Gamma ; e\right\|_{L_{p}}^{p} \leq c \operatorname{cap}_{p, t, \beta}(e), \\
& \left\|(\min \{1, y\})^{\alpha} \Gamma ; e\right\|_{L_{p}}^{p} \leq c \operatorname{cap}_{p, t-s, \beta}(e) .
\end{aligned}
$$

$$
\begin{aligned}
& \|\Gamma\|_{M\left(W_{p}^{t, \beta} \rightarrow W_{p}^{s, \alpha}\right)} \\
& \sim \sup _{\substack{e \subset \mathbb{R}_{+}^{n} \\
\operatorname{diam}(e)^{\leq} \leq 1}}\left(\frac{\left\|(\min \{1, y\})^{\alpha} \nabla_{s} \Gamma ; e\right\|_{L_{p}}}{\left(\operatorname{cap}_{p, t, \beta}(e)\right)^{1 / p}}+\frac{\left\|(\min \{1, y\})^{\alpha} \Gamma ; e\right\|_{L_{p}}}{\left(\operatorname{cap}_{p, t-s, \beta}(e)\right)^{1 / p}}\right) .
\end{aligned}
$$

An important particular case of Theorem 2 is $t=s$.

Corollary 1. A function $\Gamma$ belongs to the space $M W_{p}^{s, \alpha}$ if and only if $\Gamma \in W_{p, \text { loc }}^{s, \alpha}$ and for any compact set $e \subset \mathbb{R}_{+}^{n}$

$$
\left\|(\min \{1, y\})^{\alpha} \nabla_{s} \Gamma ; e\right\|_{L_{p}}^{p} \leq c \operatorname{cap}_{p, s, \alpha}(e)
$$

Moreover,

$$
\|\Gamma\|_{M W_{p}^{s, \alpha}} \sim \sup _{\substack{e \subset \mathbb{R}_{+}^{n} \\ \operatorname{diam}(e) \leq 1}} \frac{\left\|(\min \{1, y\})^{\alpha} \nabla_{s} \Gamma ; e\right\|_{L_{p}}}{\left(\operatorname{cap}_{p, s, \alpha}(e)\right)^{1 / p}}+\|\Gamma\|_{L_{\infty}} .
$$

\section{Trace theorems for multipliers in weighted Sobolev spaces}

We start with the following simple fact concerning traces of multipliers. 
102 Traces of multipliers in pairs of weighted Sobolev spaces

Theorem 3. Let $m$ and $l$ be positive noninteger, $m \geq l$ and let

$$
\Gamma \in M\left(W_{p}^{t, \beta}\left(\mathbb{R}_{+}^{n}\right) \rightarrow W_{p}^{s, \alpha}\left(\mathbb{R}_{+}^{n}\right)\right)
$$

where $t=[m]+1, s=[l]+1, \beta=1-\{m\}-1 / p$, and $\alpha=1-\{l\}-1 / p$. If $\gamma$ is the trace of $\Gamma$ on $\mathbb{R}^{n-1}$, then

$$
\gamma \in M\left(W_{p}^{m}\left(\mathbb{R}^{n-1}\right) \rightarrow W_{p}^{l}\left(\mathbb{R}^{n-1}\right)\right)
$$

and the estimate

$$
\left\|\gamma ; \mathbb{R}^{n-1}\right\|_{M\left(W_{p}^{m} \rightarrow W_{p}^{l}\right)} \leq c\left\|\Gamma ; \mathbb{R}_{+}^{n}\right\|_{M\left(W_{p}^{t, \beta} \rightarrow W_{p}^{s, \alpha}\right)}
$$

holds.

Proof. Let $U \in W_{p}^{t, \beta}\left(\mathbb{R}_{+}^{n}\right)$ and let $u$ be the trace of $U$ on $\mathbb{R}^{n-1}$. By setting $\Gamma U$ and $\gamma u$ instead of $U$ and $u$, respectively, in the inequality

$$
\left\|u ; \mathbb{R}^{n-1}\right\|_{W_{p}^{l}} \leq c\left\|U ; \mathbb{R}_{+}^{n}\right\|_{W_{p}^{s, \alpha}}
$$

we arrive at the estimate

$$
\left\|\gamma u ; \mathbb{R}^{n-1}\right\|_{W_{p}^{l}} \leq c\left\|\Gamma ; \mathbb{R}_{+}^{n}\right\|_{M\left(W_{p}^{t, \beta} \rightarrow W_{p}^{s, \alpha}\right)}\left\|U ; \mathbb{R}_{+}^{n}\right\|_{W_{p}^{t, \beta}} .
$$

Minimizing the right-hand side over all extensions $U$ of $u$ we obtain

$$
\left\|\gamma u ; \mathbb{R}^{n-1}\right\|_{W_{p}^{l}} \leq c\left\|\Gamma ; \mathbb{R}_{+}^{n}\right\|_{M\left(W_{p}^{t, \beta} \rightarrow W_{p}^{s, \alpha}\right)}\left\|u ; \mathbb{R}^{n-1}\right\|_{W_{p}^{m}}
$$

which gives (20).

We state an extension theorem for functions in $M\left(W_{p}^{m}\left(\mathbb{R}^{n-1}\right) \rightarrow\right.$ $\left.W_{p}^{l}\left(\mathbb{R}^{n-1}\right)\right)$ to be proved in Section 7 .

Theorem 4. Let $m$ and $l$ be positive nonintegers, $m \geq l, p \in(1, \infty)$, and let

$$
\gamma \in M\left(W_{p}^{m}\left(\mathbb{R}^{n-1}\right) \rightarrow W_{p}^{l}\left(\mathbb{R}^{n-1}\right)\right)
$$

Then the Dirichlet problem

$$
\Delta \Gamma=0 \text { on } \mathbb{R}_{+}^{n},\left.\Gamma\right|_{\mathbb{R}^{n-1}}=\gamma
$$

has a unique solution in $M\left(W_{p}^{t, \beta}\left(\mathbb{R}_{+}^{n}\right) \rightarrow W_{p}^{s, \alpha}\left(\mathbb{R}_{+}^{n}\right)\right)$, where $t=[m]+1$, $s=[l]+1, \beta=1-\{m\}-1 / p$, and $\alpha=1-\{l\}-1 / p$. There holds the estimate

$$
\left\|\Gamma ; \mathbb{R}_{+}^{n}\right\|_{M\left(W_{p}^{t, \beta} \rightarrow W_{p}^{s, \alpha}\right)} \leq c\left\|\gamma ; \mathbb{R}^{n-1}\right\|_{M\left(W_{p}^{m} \rightarrow W_{p}^{l}\right)}
$$




\section{Auxiliary estimates for an extension operator}

6.1 Pointwise estimate for $\boldsymbol{T} \gamma$ and $\boldsymbol{\nabla} \boldsymbol{T} \gamma$. For functions $\gamma \in$ $L_{1, \text { unif }}\left(\mathbb{R}^{n-1}\right)$, we introduce the operator

$$
(T \gamma)(x, y)=y^{1-n} \int_{\mathbb{R}^{n-1}} \zeta\left(\frac{x-\xi}{y}\right) \gamma(\xi) d \xi, \quad(x, y) \in \mathbb{R}_{+}^{n},
$$

where $\zeta$ is a continuously differentiable function defined on $\overline{\mathbb{R}_{+}^{n}}$ outside the origin. We assume that

$$
(|z|+1)|\nabla \zeta(z)|+|\zeta(z)| \leq C(|z|+1)^{-n} .
$$

Lemma 6. Let $\gamma \in M\left(W_{p}^{m-l}\left(\mathbb{R}^{n-1}\right) \rightarrow L_{p}\left(\mathbb{R}^{n-1}\right)\right)$, where $m \geq l$ and $1<p<\infty$. Then

$$
|T \gamma(z)|+y|\nabla(T \gamma(z))| \leq c\left(1+y^{l-m}\right)\left\|\gamma ; \mathbb{R}^{n-1}\right\|_{M\left(W_{p}^{m-l} \rightarrow L_{p}\right)} .
$$

Proof. In view of (24)

$$
\begin{aligned}
|T \gamma(z)|+ & y|\nabla(T \gamma(z))| \\
& \leq c y^{1-n}\left(\int_{\mathcal{B}_{y}^{n-1}(x)}|\gamma(\xi)| d \xi+y^{n} \int_{\mathbb{R}^{n-1} \backslash \mathcal{B}_{y}^{n-1}(x)} \frac{|\gamma(\xi)| d \xi}{|\xi-x|^{n}}\right) .
\end{aligned}
$$

By Hölder's inequality,

$$
\int_{\mathcal{B}_{y}^{n-1}(x)}|\gamma(\xi)| d \xi \leq c y^{(n-1)(p-1) / p}\left\|\gamma ; \mathcal{B}_{y}^{n-1}(x)\right\|_{L_{p}}
$$

Let $y \in(0,1)$. The right-hand side in $(26)$ does not exceed

$$
\begin{aligned}
& c y^{-m+l+n-1} \sup _{\substack{r \in(0,1) \\
x \in \mathbb{R}^{n-1}}}\left(1+r^{m-l-\frac{n-1}{p}}\right)\left\|\gamma ; \mathcal{B}_{r}^{n-1}(x)\right\|_{L_{p}} \\
& \quad \leq c y^{-m+l+n-1} \sup _{\substack{r \in(0,1) \\
x \in \mathbb{R} n-1}}\left(\operatorname{cap}_{p, m-l}\left(\mathcal{B}_{r}^{n-1}(x)\right)\right)^{-1 / p}\left\|\gamma ; \mathcal{B}_{r}^{n-1}(x)\right\|_{L_{p}} .
\end{aligned}
$$

This and Proposition 2 show that for $y \in(0,1)$

$$
\int_{\mathcal{B}_{y}^{n-1}(x)}|\gamma(\xi)| d \xi \leq c y^{-m+l+n-1}\left\|\gamma ; \mathbb{R}^{n-1}\right\|_{M\left(W_{p}^{m-l} \rightarrow L_{p}\right)} .
$$

Suppose $y>1$. Since

$$
\operatorname{cap}_{p, m-l}\left(\mathcal{B}_{r}^{n-1}(x)\right) \sim r^{n-1} \text { for } r>1,
$$


104 Traces of multipliers in pairs of weighted Sobolev spaces

it follows that the right-hand side of (26) is dominated by

$$
c y^{n-1}\left(\operatorname{cap}_{p, m-l}\left(\mathcal{B}_{y}^{n-1}(x)\right)\right)^{-1 / p}\left\|\gamma ; \mathcal{B}_{y}^{n-1}(x)\right\|_{L_{p}} .
$$

Combining this with (27) and Proposition 2 we conclude that

$$
\int_{\mathcal{B}_{y}^{n-1}(x)}|\gamma(\xi)| d \xi \leq c y^{n-1}\left(1+y^{l-m}\right)\left\|\gamma ; \mathbb{R}^{n-1}\right\|_{M\left(W_{p}^{m-l} \rightarrow L_{p}\right)} .
$$

We now estimate the second integral in the right-hand side of (25). Clearly,

$$
\int_{\mathbb{R}^{n-1} \backslash \mathcal{B}_{y}^{n-1}(x)} \frac{|\gamma(\xi)| d \xi}{|\xi-x|^{n}} \leq n \int_{y}^{\infty} \frac{d \rho}{\rho^{n+1}} \int_{\mathcal{B}_{\rho}^{n-1}(x)}|\gamma(\xi)| d \xi .
$$

By Hölder's inequality the right-hand side of (31) admits the majorant

$$
c \int_{y}^{\infty} \rho^{-2-\frac{n-1}{p}}\left\|\gamma ; \mathcal{B}_{\rho}^{n-1}(x)\right\|_{L_{p}} d \rho .
$$

Using (29) we see that the function (32), for $y>1$, does not exceed

$$
c y^{-1} \sup _{\substack{r \in(0,1) \\ x \in \mathbb{R}^{n-1}}}\left(\operatorname{cap}_{p, m-1}\left(\mathcal{B}_{r}^{n-1}(x)\right)\right)^{-1 / p}\left\|\gamma ; \mathcal{B}_{r}^{n-1}(x)\right\|_{L_{p}}
$$

which in view of Proposition 2 is dominated by

$$
c y^{-1}\left\|\gamma ; \mathbb{R}^{n-1}\right\|_{M\left(W_{p}^{m-l} \rightarrow L_{p}\right)} .
$$

Let $y<1$. Then

$$
\begin{aligned}
& \int_{y}^{1} \rho^{-2-\frac{n-1}{p}}\left\|\gamma ; \mathcal{B}_{\rho}^{n-1}(x)\right\|_{L_{p}} d \rho \\
& \leq c y^{-m+l-1} \sup _{\substack{r \in(0,1) \\
x \in \mathbb{R} n-1}}\left(1+r^{m-1-\frac{n-1}{p}}\right)\left\|\gamma ; \mathcal{B}_{r}^{n-1}(x)\right\|_{L_{p}} \\
& \quad \leq c y^{-m+l-1} \sup _{\substack{r \in(0,1) \\
x \in \mathbb{R}^{n-1}}}\left(\operatorname{cap}_{p, m-l}\left(\mathcal{B}_{r}^{n-1}(x)\right)\right)^{-1 / p}\left\|\gamma ; \mathcal{B}_{r}^{n-1}(x)\right\|_{L_{p}} .
\end{aligned}
$$

Furthermore, by (29) 


$$
\begin{aligned}
\int_{1}^{\infty} \rho^{-2-\frac{n-1}{p}}\left\|\gamma ; \mathcal{B}_{\rho}^{n-1}(x)\right\|_{L_{p}} d \rho \\
\quad \leq c \int_{1}^{\infty} \rho^{-2}\left(\operatorname{cap}_{p, m-l}\left(\mathcal{B}_{\rho}^{n-1}(x)\right)\right)^{-1 / p}\left\|\gamma ; \mathcal{B}_{\rho}^{n-1}(x)\right\|_{L_{p}} d \rho \\
\quad \leq c \sup _{\substack{r>0 \\
x \in \mathbb{R}^{n-1}}}\left(\operatorname{cap}_{p, m-l}\left(\mathcal{B}_{r}^{n-1}(x)\right)\right)^{-1 / p}\left\|\gamma ; \mathcal{B}_{r}^{n-1}(x)\right\|_{L_{p}} .
\end{aligned}
$$

Summing up this inequality and (34), and using Proposition 2 we conclude that the integral (32) is majorized, for $y<1$, by

$$
c y^{-m+l-1}\left\|\gamma ; \mathbb{R}^{n-1}\right\|_{M\left(W_{p}^{m-l} \rightarrow L_{p}\right)} .
$$

This, together with (33), imply that for all $y>0$ the integral (32) does not exceed

$$
c y^{-1}\left(1+y^{l-m}\right)\left\|\gamma ; \mathbb{R}^{n-1}\right\|_{M\left(W_{p}^{m-l} \rightarrow L_{p}\right)} .
$$

Hence, the result follows from (30), (31), and (25).

\subsection{Weighted $L_{p}$-estimates for $T \gamma$ and $\nabla T \gamma$}

Lemma 7. Let the extension operator $T$ be defined by (23) and suppose that $\gamma \in M\left(W_{p}^{m-l}\left(\mathbb{R}^{n-1}\right) \rightarrow L_{p}\left(\mathbb{R}^{n-1}\right)\right)$, where $l \in(0,1),[m] \geq 1,1<p<$ $\infty$. Then, for $k=1, \ldots,[m]$,

$$
\begin{gathered}
\left(\int_{0}^{1} y^{p(k-l)-1}(|T \gamma(z)|+y|\nabla(T \gamma)(z)|)^{p} d y\right)^{1 / p} \\
\quad \leq c\left\|\gamma ; \mathbb{R}^{n-1}\right\|_{M\left(W_{p}^{m-l} \rightarrow L_{p}\right)}^{\frac{k-l}{m-l}}[(\mathcal{M} \gamma)(x)]^{\frac{m-k}{m-l}},
\end{gathered}
$$

where $\mathcal{M}$ is the Hardy-Littlewood maximal operator in $\mathbb{R}^{n-1}$.

Proof. Let $\delta$ be a number in $(0,1]$ to be chosen later. We set

$$
\int_{0}^{1} y^{p(k-l)-1}(|T \gamma|+y|\nabla(T \gamma)(z)|)^{p} d y=\int_{0}^{\delta} \ldots d y+\int_{\delta}^{1} \ldots d y
$$

In view of $(25)$

$$
\int_{0}^{\delta} \ldots d y \leq c \int_{0}^{\delta} y^{p(k+1-l-n)-1}\left(\int_{\mathcal{B}_{y}^{n-1}(x)}|\gamma(\xi)| d \xi\right)^{p} d y
$$




$$
+c \int_{0}^{\delta} y^{p(k+1-l)-1}\left(\int_{\mathbb{R}^{n-1} \backslash \mathcal{B}_{y}^{n-1}(x)} \frac{|\gamma(\xi)| d \xi}{|\xi-x|^{n}}\right)^{p} d y .
$$

By the definition of $\mathcal{M}$,

$$
\int_{0}^{\delta} y^{p(k+1-l-n)-1}\left(\int_{\mathcal{B}_{y}^{n-1}(x)}|\gamma(\xi)| d \xi\right)^{p} d y \leq c[(\mathcal{M} \gamma)(x)]^{p} \delta^{p(k-l)} .
$$

Using (31) we obtain

$$
\int_{0}^{\delta} y^{p(k+1-l)-1}\left(\int_{\mathbb{R}^{n-1} \backslash \mathcal{B}_{y}^{n-1}(x)} \frac{|\gamma(\xi)| d \xi}{|\xi-x|^{n}}\right)^{p} d y \leq c[(\mathcal{M} \gamma)(x)]^{p} \delta^{p(k-l)}
$$

Combining (36) and (37) we conclude that

$$
\int_{0}^{\delta} \ldots d y \leq c[(\mathcal{M} \gamma)(x)]^{p} \delta^{p(k-l)}
$$

By Lemma 6,

$$
\begin{aligned}
& \int_{\delta}^{1} y^{p(k-l)-1}(|T \gamma|+y|\nabla(T \gamma)(z)|)^{p} d y \\
& \quad \leq c\left\|\gamma ; \mathbb{R}^{n-1}\right\|_{M\left(W_{p}^{m-l} \rightarrow L_{p}\right)}^{p} \delta^{p(k-m)} .
\end{aligned}
$$

Summing up (38) and (39) we find

$$
\begin{aligned}
& \int_{0}^{1} y^{p(k-l)-1}(|T \gamma|+y|\nabla(T \gamma)(z)|)^{p} d y \\
& \quad \leq c\left([(\mathcal{M} \gamma)(x)]^{p} \delta^{p(k-l)}+\left\|\gamma ; \mathbb{R}^{n-1}\right\|_{M\left(W_{p}^{m-l} \rightarrow L_{p}\right)}^{p} \delta^{p(k-m)}\right) .
\end{aligned}
$$

The right-hand side in this inequality attains its minimum value for

$$
\delta=\left(\frac{\left\|\gamma ; \mathbb{R}^{n-1}\right\|_{M\left(W_{p}^{m-l} \rightarrow L_{p}\right)}}{(\mathcal{M} \gamma)(x)}\right)^{1 /(m-l)} .
$$

The proof is complete.

Lemma 8. Let the operators $T$ and $D_{p, l}$ be defined by (23) and (2). Then

$$
\int_{0}^{1} y^{p(1-l)-1}|\nabla(T \gamma)(z)|^{p} d y \leq c\left(\left(D_{p, l} \gamma\right)(x)\right)^{p} .
$$


Proof. Let $R(\xi, x)=\gamma(\xi)-\gamma(x)$. Using the identity

$$
y^{-n+1} \int_{\mathbb{R}^{n-1}} \zeta\left(\frac{\xi-x}{y}\right) d \xi=\text { const }
$$

we have

$$
\frac{\partial T \gamma}{\partial y}(x, y)=\frac{\partial}{\partial y}\left(y^{-n+1} \int_{\mathbb{R}^{n-1}} \zeta\left(\frac{\xi-x}{y}\right) R(\xi, x) d \xi\right) .
$$

Furthermore, it is clear that

$$
\frac{\partial T \gamma}{\partial x_{j}}(x, y)=y^{-n+1} \int_{\mathbb{R}^{n-1}} R(\xi, x) \frac{\partial}{\partial x_{j}} \zeta\left(\frac{\xi-x}{y}\right) d \xi .
$$

Therefore,

$$
|\nabla(T \gamma)(x, y)| \leq c y^{-n} \sum_{k=0}^{1} \int_{\mathbb{R}^{n-1}}\left|\nabla_{k} \zeta\left(\frac{\xi-x}{y}\right)\right|\left(1+\frac{|\xi-x|}{y}\right)^{k}|R(\xi, x)| d \xi .
$$

This estimate and (24) imply

$$
\begin{aligned}
& |\nabla(T \gamma)(x, y)| \leq c y^{-n} \int_{\mathbb{R}^{n-1}}\left(1+\frac{|\xi-x|}{y}\right)^{-n}|R(\xi, x)| d \xi \\
& \quad=c y^{-1 / p} \int_{\mathbb{R}^{n-1}}\left(\frac{|\xi-x|}{y}\right)^{n-1 / p}\left(1+\frac{|\xi-x|}{y}\right)^{-n} \frac{|R(\xi, x)|}{|\xi-x|^{n-1 / p}} d \xi .
\end{aligned}
$$

Consequently,

$$
\begin{aligned}
& \int_{0}^{1} y^{p(1-l)-1}|\nabla(T \gamma)(x, y)|^{p} d y \\
& \quad \leq c \int_{0}^{1}\left(\int_{\mathbb{R}^{n-1}} f\left(\frac{|\xi-x|}{y}\right) \frac{|R(\xi, x)|}{|\xi-x|^{n-1 / p}} d \xi\right)^{p} y^{p(1-l)-1} \frac{d y}{y},
\end{aligned}
$$

where $f(\eta)=\eta^{n-1 / p}(1+\eta)^{-n}$. We write the last integral over $(0,1)$ as

$$
\begin{aligned}
& \int_{0}^{1}\left(\int_{0}^{\infty} f\left(\frac{t}{y}\right) g(t, x) \frac{d t}{t}\right)^{p} y^{p(1-l)-1} \frac{d y}{y} \\
& \quad=\int_{0}^{1}\left(\int_{0}^{\infty} f(s) g(s y, x) \frac{d s}{s}\right)^{p} y^{p(1-l)-1} \frac{d y}{y}
\end{aligned}
$$


with

$$
g(t, x)=t^{1 / p-1} \int_{\partial \mathcal{B}_{1}^{n-1}}|R(t \theta+x, x)| d \theta .
$$

By Minkowski's inequality, the right-hand side of (41) does not exceed

$$
\begin{aligned}
& \left(\int_{0}^{\infty}\left(\int_{0}^{1}(f(s))^{p}(g(s y, x))^{p} y^{p(1-l)-1} \frac{d y}{y}\right)^{1 / p} \frac{d s}{s}\right)^{p} \\
& =\left(\int_{0}^{\infty} f(s)\left(\int_{0}^{s}(g(\tau, x))^{p} \tau^{p(1-l)-1} \frac{d \tau}{\tau}\right)^{1 / p} \frac{d s}{s^{2-l-1 / p}}\right)^{p} \\
& \leq\left(\int_{0}^{\infty} f(s) \frac{d s}{s^{2-l-1 / p}}\right)^{p} \int_{0}^{\infty}(g(\tau, x))^{p} \tau^{p(1-l)-1} \frac{d \tau}{\tau} .
\end{aligned}
$$

Therefore,

$$
\int_{0}^{1} y^{p(1-l)-1}|\nabla(T \gamma)(x, y)|^{p} d y \leq c \int_{0}^{\infty}(g(\tau, x))^{p} \tau^{p(1-l)-1} \frac{d \tau}{\tau} .
$$

It remains to note that

$$
\begin{aligned}
\int_{0}^{\infty}(g(\tau, x))^{p} \tau^{p(1-l)-1} \frac{d \tau}{\tau} & =\int_{0}^{\infty} \tau^{-p l}\left(\int_{\partial \mathcal{B}_{1}^{n-1}}|\gamma(\tau \theta+x)-\gamma(x)| d \theta\right)^{p} \frac{d \tau}{\tau} \\
& \leq c \int_{0}^{\infty} \int_{\partial \mathcal{B}_{1}^{n-1}}|\gamma(\tau \theta+x)-\gamma(x)|^{p} d \theta \frac{d \tau}{\tau^{p l+1}} \\
& \leq c \int_{\mathbb{R}^{n-1}} \frac{|\gamma(x+h)-\gamma(x)|^{p}}{|h|^{p l+n-1}} d h \\
& =c\left(\left(D_{p, l} \gamma\right)(x)\right)^{p} .
\end{aligned}
$$

The result follows.

\section{Proof of Theorem 4}

7.1 The case $l<\mathbf{1}$. Our aim now is to prove that for $l<1$ and $s=1$ the operator $T$ defined by (23) maps $M\left(W_{p}^{m}\left(\mathbb{R}^{n-1}\right) \rightarrow W_{p}^{l}\left(\mathbb{R}^{n-1}\right)\right)$ into $M\left(W_{p}^{[m]+1, \beta}\left(\mathbb{R}_{+}^{n}\right) \rightarrow W_{p}^{1, \alpha}\left(\mathbb{R}_{+}^{n}\right)\right)$ with $\alpha=1-l-1 / p, \beta=1-\{m\}-1 / p$ and there holds the estimate

$$
\left\|T \gamma ; \mathbb{R}_{+}^{n}\right\|_{M\left(W_{p}^{t, \beta} \rightarrow W_{p}^{1, \alpha}\right)} \leq c C\left\|\gamma ; \mathbb{R}^{n-1}\right\|_{M\left(W_{p}^{m} \rightarrow W_{p}^{l}\right)},
$$

where $C$ is the constant in (24). 
We have

$$
\begin{aligned}
& \begin{aligned}
\left\|(\min \{1, y\})^{\alpha} \nabla(U T \gamma) ; \mathbb{R}_{+}^{n}\right\|_{L_{p}}^{p} \leq c \int_{0}^{1} y^{p \alpha} \int_{\mathbb{R}^{n-1}}\left(|\nabla(T \gamma)|^{p}|U|^{p}+|T \gamma|^{p}|\nabla U|^{p}\right) d z \\
+c \int_{1}^{\infty} \int_{\mathbb{R}^{n-1}}\left(|\nabla(T \gamma)|^{p}|U|^{p}+|T \gamma|^{p}|\nabla U|^{p}\right) d z \\
=c \int_{0<y<1} \ldots d z+c \int_{y>1} \ldots d z .
\end{aligned}
\end{aligned}
$$

By Lemma 1, for $y>1$

Hence,

$$
y|\nabla(T \gamma)(z)|+|(T \gamma)(z)| \leq c\left\|\gamma ; \mathbb{R}^{n-1}\right\|_{M\left(W_{p}^{m-l} \rightarrow L_{p}\right)} .
$$

$$
\int_{y>1} \ldots d z \leq c\left\|\gamma ; \mathbb{R}^{n-1}\right\|_{M\left(W_{p}^{m-l} \rightarrow L_{p}\right)}^{p}\left\|U ; \mathbb{R}_{+}^{n}\right\|_{W_{p}^{1, \alpha}}^{p} .
$$

It remains to refer to the estimate

$$
\left\|U ; \mathbb{R}_{+}^{n}\right\|_{W_{p}^{1, \alpha}} \leq c\left\|U ; \mathbb{R}_{+}^{n}\right\|_{W_{p}^{t, \beta}}
$$

which follows from the one dimensional Hardy inequality.

Introducing the notation

$$
\begin{aligned}
& \mathcal{R}_{0} U(z)=U(z)-\sum_{k=0}^{[m]} \frac{\partial^{k}}{\partial y^{k}} U(x, 0) \frac{y^{k}}{k !}, \\
& \mathcal{R}_{1} U(z)= \begin{cases}\nabla U(z)-\sum_{k=0}^{[m]-1} \frac{\partial^{k}}{\partial y^{k}} \nabla U(x, 0) \frac{y^{k}}{k !} & \text { for } m>1 \\
\nabla U(z) & \text { for } m<1\end{cases}
\end{aligned}
$$

we have

$$
\begin{gathered}
\int_{0<y<1} \ldots d z \leq c \int_{0<y<1} y^{p(1-l)-1} \sum_{j=0}^{1}\left|\nabla_{j}(T \gamma)\right|^{p}\left|\mathcal{R}_{1-j} U(z)\right|^{p} d z \\
+c \int_{0<y<1} y^{-p l-1}(|T \gamma(z)|+y|\nabla(T \gamma)(z)|)^{p} \sum_{k=1}^{[m]}\left|\nabla_{k} U(x, 0)\right|^{p} y^{p k} d z \\
+c \int_{0<y<1} y^{p(1-l)-1}|\nabla T \gamma(z)|^{p}|U(x, 0)|^{p} d z
\end{gathered}
$$


for $m>1$. In case $m<1$ the second integral in the right hand side of (46) should be omitted.

By Lemma 6, for $0<y<1$

$$
|T \gamma(z)|+y|\nabla(T \gamma)(z)| \leq c y^{l-m}\left\|\gamma ; \mathbb{R}^{n-1}\right\|_{M\left(W_{p}^{m-l} \rightarrow L_{p}\right)} .
$$

Since for $j=0,1$

$$
\left|\mathcal{R}_{1-j} U(z)\right| \leq \frac{y^{[m]+j-1}}{([m]+j-1) !} \int_{0}^{y}\left|\nabla_{t} U(x, t)\right| d t
$$

we have

$$
\begin{gathered}
\int_{0<y<1} y^{p(1-\{m\})-1}\left|\mathcal{R}_{1-j} U(z)\right|^{p} d z \\
\leq c \int_{0<y<1} y^{-p\{m\}-1}\left(\int_{0}^{y}\left|\nabla_{[m]+1} U(x, t)\right| d t\right)^{p} d z .
\end{gathered}
$$

By Hardy's inequality the right-hand side does not exceed $c\left\|U ; \mathbb{R}_{+}^{n}\right\|_{W_{p}^{[m]+1, \beta}}^{p}$. Combining this with (47) we obtain that the first integral in the right-hand side of (46) does not exceed

$$
\left\|\gamma ; \mathbb{R}^{n-1}\right\|_{M\left(W_{p}^{m-l} \rightarrow L_{p}\right)}^{p}\left\|U ; \mathbb{R}_{+}^{n}\right\|_{W_{p}^{[m]+1, \beta}}^{p} .
$$

We now pass to the estimate of the second integral in the right-hand side of (46) for $k=1, \ldots,[m], m>1$. Applying Lemma 7 , we find

$$
\begin{aligned}
& \int_{0<y<1} y^{p(k-l)-1}(|T \gamma(z)|+y|\nabla(T \gamma)(z)|)^{p}\left|\nabla_{k} U(x, 0)\right|^{p} d z \\
& \leq c\left\|\gamma ; \mathbb{R}^{n-1}\right\|_{M\left(W_{p}^{m-l} \rightarrow L_{p}\right)}^{p \frac{k-l}{m-l}} \int_{\mathbb{R}^{n-1}}(\mathcal{M} \gamma(x))^{p \frac{m-k}{m-l}}\left|\nabla_{k} U(x, 0)\right|^{p} d x .
\end{aligned}
$$

The last integral is not greater than

$$
\left\|(\mathcal{M} \gamma)^{\frac{m-k}{m-l}} ; \mathbb{R}^{n-1}\right\|_{M\left(W_{p}^{m-k} \rightarrow L_{p}\right)}^{p}\left\|\nabla_{k} U(\cdot, 0) ; \mathbb{R}^{n-1}\right\|_{W_{p}^{m-k}}^{p} .
$$

Using Proposition 2 with $\lambda=m-k, \mu=m-l$ and Verbitsky's theorem on the boundedness of the maximal operator $\mathcal{M}$ in the space $M\left(W_{p}^{m-l}\left(\mathbb{R}^{n-1}\right) \rightarrow L_{p}\left(\mathbb{R}^{n-1}\right)\right)$ (see [3], Ch.2), we find that (51) is dominated by

$$
c\left\|\gamma, \mathbb{R}^{n-1}\right\|_{M\left(W_{p}^{m-l} \rightarrow L_{p}\right)}^{\frac{p(m-k)}{m-l}}\left\|U(\cdot, 0) ; \mathbb{R}^{n-1}\right\|_{W_{p}^{m}}^{p} .
$$


Hence and by (50)

$$
\begin{gathered}
\int_{0<y<1} y^{p(k-l)-1}(|T \gamma(z)|+y|\nabla(T \gamma)(z)|)^{p}\left|\nabla_{k} U(x, 0)\right|^{p} d z \\
\leq c\left\|\gamma, \mathbb{R}^{n-1}\right\|_{M\left(W_{p}^{m-l} \rightarrow L_{p}\right)}^{p}\left\|U ; \mathbb{R}_{+}^{n}\right\|_{W_{p}^{[m]+1, \beta}}^{p} .
\end{gathered}
$$

By Lemma 8, the integral

$$
\int_{0<y<1} y^{p(1-l)-1}|\nabla(T \gamma)(z)|^{p}|U(x, 0)|^{p} d z
$$

does not exceed

$$
\begin{aligned}
c \int_{\mathbb{R}^{n-1}} & \left(D_{p, l} \gamma(x)\right)^{p}|U(x, 0)|^{p} d x \\
\leq & c\left\|D_{p, l} \gamma ; \mathbb{R}^{n-1}\right\|_{M\left(W_{p}^{m} \rightarrow L_{p}\right)}^{p}\left\|U(\cdot, 0) ; \mathbb{R}^{n-1}\right\|_{W_{p}^{m}}^{p} \\
\leq & c\left\|\gamma ; \mathbb{R}^{n-1}\right\|_{M\left(W_{p}^{m} \rightarrow W_{p}^{l}\right)}^{p}\left\|U ; \mathbb{R}_{+}^{n}\right\|_{W_{p}^{[m]+1, \beta}}^{p} .
\end{aligned}
$$

Thus we arrive at the inequality

$$
\int_{0<y<1} y^{p \alpha}|\nabla(U T \gamma)(z)|^{p} d z \leq c\left\|\gamma ; \mathbb{R}^{n-1}\right\|_{M\left(W_{p}^{m} \rightarrow W_{p}^{l}\right)}^{p}\left\|U ; \mathbb{R}_{+}^{n}\right\|_{W_{p}^{[m]+1, \beta}}^{p} .
$$

It remains to estimate the integral

$$
\int_{0<y<1} y^{p(1-l)-1}|(T \gamma)(z)|^{p}|U(z)|^{p} d z .
$$

Clearly,

$$
\begin{aligned}
& \int_{0<y<1} y^{p(1-l)-1}|(T \gamma)(z)|^{p}|U(z)|^{p} d z \leq \int_{0<y<1} y^{p(1-l)-1}|(T \gamma)(z)|^{p}\left|\mathcal{R}_{0} U(z)\right|^{p} d z \\
& (55) \quad+\sum_{k=0}^{[m]} \int_{0<y<1} y^{p k} y^{p(1-l)-1}|(T \gamma)(z)|^{p}\left|\nabla_{k} U(x, 0)\right|^{p} d z
\end{aligned}
$$


112 Traces of multipliers in pairs of weighted Sobolev spaces

By (47) and (48) with $j=1$ we have

$$
\begin{aligned}
& \int_{0<y<1} y^{p(1-l)-1}|(T \gamma)(z)|^{p}\left|\mathcal{R}_{0} U(z)\right|^{p} d z \\
& \leq c\left\|\gamma ; \mathbb{R}^{n-1}\right\|_{M\left(W_{p}^{m-l} \rightarrow L_{p}\right)}^{p} \\
& \quad \times \int_{0<y<1} y^{p(1-\{m\})-1}\left(\int_{0}^{y}\left|\nabla_{[m]+1} U(x, t)\right| d t\right)^{p} d z
\end{aligned}
$$

which by Hardy's inequality is dominated by (49). In view of (52)

$$
\begin{aligned}
& \int_{0<y<1} y^{p(k-l)-1}|(T \gamma)(z)|^{p}\left|\nabla_{k} U(x, 0)\right|^{p} d z \\
& \quad \leq c\left\|\gamma ; \mathbb{R}^{n-1}\right\|_{M\left(W_{p}^{m-l} \rightarrow L_{p}\right)}^{p}\left\|U ; \mathbb{R}_{+}^{n}\right\|_{W_{p}^{[m]+1, \beta}}^{p} .
\end{aligned}
$$

Thus we arrive at the estimate

$$
\int_{0<y<1} \ldots d z \leq c\left\|\gamma ; \mathbb{R}^{n-1}\right\|_{M\left(W_{p}^{m} \rightarrow W_{p}^{l}\right)}^{p}\left\|U ; \mathbb{R}_{+}^{n}\right\|_{W_{p}^{[m]+1, \beta}}^{p} .
$$

Since the Poisson kernel satisfies condition (24), Theorem 4 with $l<1$ follows.

\subsection{The case $l>1$.}

Lemma 9. Let $m$ and $l$ be nonintegers, $m \geq l>0$, and let $T$ be the extension operator (23). Suppose that $\gamma \in M\left(W_{p}^{m-l}\left(\mathbb{R}^{n-1}\right) \rightarrow L_{p}\left(\mathbb{R}^{n-1}\right)\right)$. Then

$$
T \gamma \in M\left(W_{p}^{[m]-[l], \beta}\left(\mathbb{R}_{+}^{n}\right) \rightarrow W_{p}^{0, \alpha}\left(\mathbb{R}_{+}^{n}\right)\right)
$$

and

$$
\left\|T \gamma ; \mathbb{R}_{+}^{n}\right\|_{M\left(W_{p}^{[m]-[l], \beta} \rightarrow W_{p}^{0, \alpha}\right)} \leq c\left\|\gamma ; \mathbb{R}^{n-1}\right\|_{M\left(W_{p}^{m-l} \rightarrow L_{p}\right)} .
$$

Proof. To begin with, let $[m]=[l]$. Then by $(47)$

$$
\begin{aligned}
& \int_{0<y<1} y^{p(1-\{l\})-1}|U(z)(T \gamma)(z)|^{p} d z \\
& \quad \leq c\left\|\gamma ; \mathbb{R}^{n-1}\right\|_{M\left(W_{p}^{m-l} \rightarrow L_{p}\right)}^{p} \int_{0<y<1} y^{p(1-\{m\})-1}|U(z)|^{p} d z
\end{aligned}
$$

which gives the result. 
Suppose $[m] \geq[l]+1$. We introduce the function

$$
\mathcal{R} U=U(z)-\sum_{j=0}^{[m]-[l]-1} \frac{\partial^{j} U}{\partial y^{j}}(x, 0) \frac{y^{j}}{j !}
$$

which, clearly, satisfies

$$
|\mathcal{R} U(z)| \leq \frac{y^{[m]-[l]-1}}{([m]-[l]-1) !} \int_{0}^{y}\left|\nabla_{[m]-[l]} U(x, t)\right| d t .
$$

This and (47) imply

$$
\begin{aligned}
& \int_{0<y<1} y^{p(1-\{l\})-1}|T \gamma(z)|^{p}|\mathcal{R} U(z)|^{p} d z \\
& \leq c\left\|\gamma ; \mathbb{R}^{n-1}\right\|_{M\left(W_{p}^{m-l} \rightarrow L_{p}\right)}^{p} \int_{0<y<1} y^{-p\{m\}-1}\left(\int_{0}^{y}\left|\nabla_{[m]-[l]} U(x, t)\right| d t\right)^{p} d z .
\end{aligned}
$$

By Hardy's inequality the right-hand side is dominated by

$$
c\left\|\gamma ; \mathbb{R}^{n-1}\right\|_{M\left(W_{p}^{m-l} \rightarrow L_{p}\right)}^{p}\left\|U ; \mathbb{R}_{+}^{n}\right\|_{W_{p}^{[m]-[l], \beta}}^{p} .
$$

Furthermore, by Lemma 7 with $m$ replaced by $m-[l], l$ replaced by $\{l\}$ and $k=j+1$ we have for $j=0, \ldots,[m]-[l]-1$

$$
\begin{aligned}
& \int_{0<y<1} y^{p(j+1-\{l\})-1}|T \gamma(z)|^{p}\left|\nabla_{j} U(x, 0)\right|^{p} d z \\
& (57) \leq c\left\|\gamma ; \mathbb{R}^{n-1}\right\|_{M\left(W_{p}^{m-l} \rightarrow L_{p}\right)}^{\frac{j+1-\{l\}}{m-l}} \int_{\mathbb{R}^{n-1}}(\mathcal{M} \gamma(x))^{\frac{p-[l]-j-1}{m-l}}\left|\nabla_{j} U(x, 0)\right|^{p} d x .
\end{aligned}
$$

The last integral is dominated by

$$
\left\|(\mathcal{M} \gamma)^{\frac{p-[l]-j-1}{m-l}} ; \mathbb{R}^{n-1}\right\|_{M\left(W_{p}^{m-[l]-j-1} \rightarrow L_{p}\right)}^{p}\left\|U(\cdot, 0) ; \mathbb{R}^{n-1}\right\|_{W_{p}^{m-[l]-1}}^{p}
$$

which by Proposition 3 does not exceed

$$
\left\|\mathcal{M} \gamma ; \mathbb{R}^{n-1}\right\|_{M\left(W_{p}^{m-l} \rightarrow L_{p}\right)}^{p \frac{m-[l]-j-1}{m-l}}\left\|U ; \mathbb{R}_{+}^{n}\right\|_{W_{p}^{[m]-[l], \beta}}^{p} .
$$

Hence and by (57)

$$
\int_{0<y<1} y^{p(j+1-\{l\})-1}|T \gamma(z)|^{p}\left|\nabla_{j} U(x, 0)\right|^{p} d z
$$




$$
\leq c\left\|\gamma ; \mathbb{R}^{n-1}\right\|_{M\left(W_{p}^{m-l} \rightarrow L_{p}\right)}^{p}\left\|U ; \mathbb{R}_{+}^{n}\right\|_{W_{p}^{[m]-[l], \beta}}^{p} .
$$

The result follows.

Proof of Theorem 4 for $l>1$. Suppose Theorem has been proved for $[l]=1, \ldots, \mathcal{L}-1$, where $\mathcal{L} \geq 2$. Let $[l]=\mathcal{L}$ and let

$$
\gamma \in M\left(W_{p}^{m}\left(\mathbb{R}^{n-1}\right) \rightarrow W_{p}^{l}\left(\mathbb{R}^{n-1}\right)\right) \text { for } m \geq \mathcal{L} .
$$

Let $T \gamma$ denote the Poisson integral. Since by Proposition 1 one has

$$
\gamma \in M\left(W_{p}^{m-l}\left(\mathbb{R}^{n-1}\right) \rightarrow L_{p}\left(\mathbb{R}^{n-1}\right)\right),
$$

it follows from Lemma 9 that

$$
T \gamma \in M\left(W_{p}^{[m]-[l], \beta}\left(\mathbb{R}_{+}^{n}\right) \rightarrow W_{p}^{0, \alpha}\left(\mathbb{R}_{+}^{n}\right)\right)
$$

and (56) holds. Next we show that

$$
\nabla_{\mathcal{L}+1}(T \gamma) \in M\left(W_{p}^{[m]+1, \beta}\left(\mathbb{R}_{+}^{n}\right) \rightarrow W_{p}^{0, \alpha}\left(\mathbb{R}_{+}^{n}\right)\right) .
$$

Using Proposition 1, we obtain

$$
\frac{\partial \gamma}{\partial x_{k}} \in M\left(W_{p}^{m}\left(\mathbb{R}^{n-1}\right) \rightarrow W_{p}^{l-1}\left(\mathbb{R}^{n-1}\right)\right), \quad k=1, \ldots, n-1 .
$$

Then, by the induction hypothesis applied to $\partial \gamma / \partial x_{k}$,

$$
\frac{\partial}{\partial x_{k}}(T \gamma)=T \frac{\partial \gamma}{\partial x_{k}} \in M\left(W_{p}^{[m]+1, \beta}\left(\mathbb{R}_{+}^{n}\right) \rightarrow W_{p}^{\mathcal{L}, \alpha}\left(\mathbb{R}_{+}^{n}\right)\right) .
$$

By Lemma 3,

$$
\nabla_{\mathcal{L}} \frac{\partial}{\partial x_{k}}(T \gamma) \in M\left(W_{p}^{[m]+1, \beta}\left(\mathbb{R}_{+}^{n}\right) \rightarrow W_{p}^{0, \alpha}\left(\mathbb{R}_{+}^{n}\right)\right) .
$$

Using the harmonicity of $T \gamma$ and (61) we find

$$
\frac{\partial^{\mathcal{L}+1}(T \gamma)}{\partial y^{\mathcal{L}+1}}=-\frac{\partial^{\mathcal{L}-1}\left(\Delta_{x}(T \gamma)\right)}{\partial y^{\mathcal{L}-1}} \in M\left(W_{p}^{[m]+1, \beta}\left(\mathbb{R}_{+}^{n}\right) \rightarrow W_{p}^{0, \alpha}\left(\mathbb{R}_{+}^{n}\right)\right)
$$

which together with (61) implies the inclusion (59). Combining this with (56) we find that $T \gamma \in M\left(W_{p}^{[m]+1, \beta}\left(\mathbb{R}_{+}^{n}\right) \rightarrow W_{p}^{[l]+1, \alpha}\left(\mathbb{R}_{+}^{n}\right)\right)$. It remains to note that all above inclusions are accompanied by the corresponding estimates. The result follows. 


\section{Extension of multipliers defined on $\partial \Omega$}

We return to the assertion stated in Introduction.

Theorem 5. Let $\gamma \in M\left(W_{p}^{m}(\partial \Omega) \rightarrow W_{p}^{l}(\partial \Omega)\right)$, where $m$ and $l$ are nonintegers, $m \geq l>0, p \in(1, \infty)$. There exists a linear extension operator

$$
\gamma \rightarrow \Gamma \in M\left(W_{p}^{t, \beta}(\Omega) \rightarrow W_{p}^{s, \alpha}(\Omega)\right),
$$

where $t=[m]+1, s=[l]+1, \beta=1-\{m\}-1 / p$, and $\alpha=1-\{l\}-1 / p$.

Proof. It suffices to construct an extension $\Gamma$ only for $\gamma$ with sufficiently small support. To be precise, we assume that $\gamma=0$ outside the ball $\mathcal{B}_{\rho}^{n}$ centered at $0 \in \partial \Omega$, where $\rho$ is small enough. We introduce a cut off function $\varphi \in C_{0}^{\infty}\left(\mathcal{B}_{3 \rho}^{n}\right)$, equal to one on $\mathcal{B}_{2 \rho}^{n}$. Let us define cartesian coordinates $\zeta=(\xi, \eta)$ with the origin 0 , where $\xi \in \mathbb{R}^{n-1}$ and $\eta \in \mathbb{R}^{1}$. Let $\Omega \cap \mathcal{B}_{3 \rho}^{n}=\left\{\zeta: \xi \in \mathcal{B}_{3 \rho}^{n-1}, \eta>f(\xi)\right\}$, where $f$ is a smooth function. We make the standard change of variables $\kappa: \zeta \rightarrow(x, y)$, where $x=\xi$, $y=\eta-f(\xi)$. The diffeomorphism $\kappa$ maps $\Omega \cap \mathcal{B}_{3 \rho}^{n}$ into the half space $\mathbb{R}_{+}^{n}=\left\{(x, y): x \in \mathbb{R}^{n-1}, y>0\right\}$. Clearly, the function $\tilde{\gamma}=\gamma \circ \kappa^{-1}$ belongs to $M\left(W_{p}^{m}\left(\mathbb{R}^{n-1}\right) \rightarrow W_{p}^{l}\left(\mathbb{R}^{n-1}\right)\right)$. Its harmonic extension to $\mathbb{R}_{+}^{n}$, denoted by $\tilde{\Gamma}$, is in $M\left(W_{p}^{t, \beta}\left(\mathbb{R}_{+}^{n}\right) \rightarrow W_{p}^{s, \alpha}\left(\mathbb{R}_{+}^{n}\right)\right)$ and satisfies the estimate (22) according to Theorem 4. Hence the function $\gamma=(\tilde{\Gamma} \circ \kappa) \varphi$ is a desired extension. The proof is complete.

\section{References}

[1] K. F. Andersen, Weighted inequalities for convolutions, Proc. Amer. Math. Soc., 123 (4) (1995), 1129-1136.

[2] V. Maz'ya, Sobolev Spaces, Springer, 1985.

[3] V. Maz'ya and T. Shaposhnikova, Theory of Multipliers in Spaces of Differentiable Functions, Pitman, 1985.

[4] H. Triebel, Interpolation Theory, Function Spaces, Differential Operators, VEB Deutscher Verlag der Wiss., Berlin, 1978.

[5] S. V. Uspenskii, Imbedding theorems for classes with weights, Tr. Mat. Inst. Steklova, 60 (1961), 282-303 (Russian); English translation: Amer. Math. Soc. Transl., 87 (1970), 121-145.

Department of Mathematics, University of Linköping

SE-581 83 Linköping, Sweden

(E-mail : vlmaz@mai.liu.se)

(E-mail :tasha@mai.liu.se)

(Received : April 2004) 


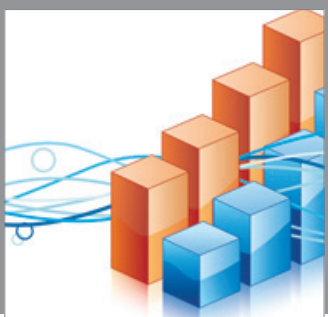

Advances in

Operations Research

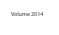

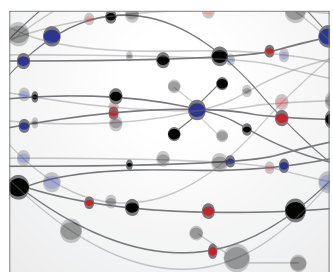

\section{The Scientific} World Journal
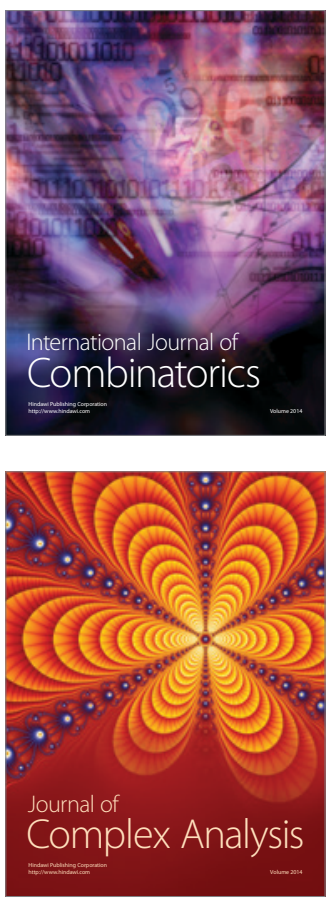

International Journal of

Mathematics and

Mathematical

Sciences
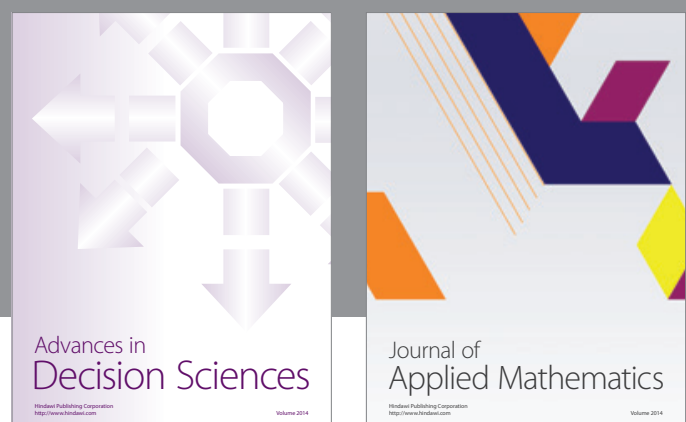

Journal of

Applied Mathematics
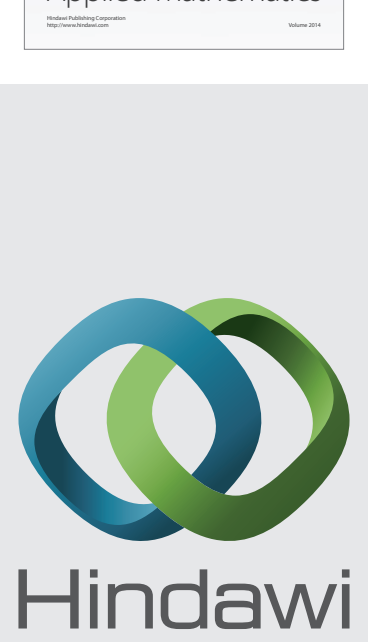

Submit your manuscripts at http://www.hindawi.com
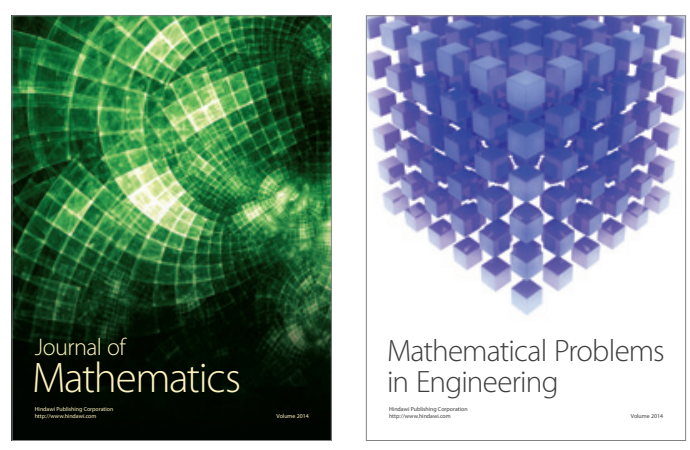

Mathematical Problems in Engineering
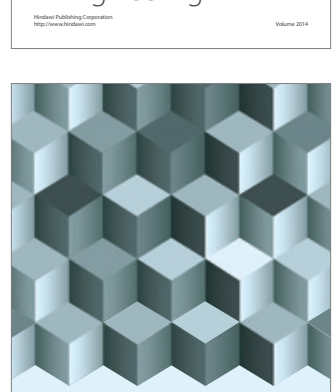

Journal of

Function Spaces
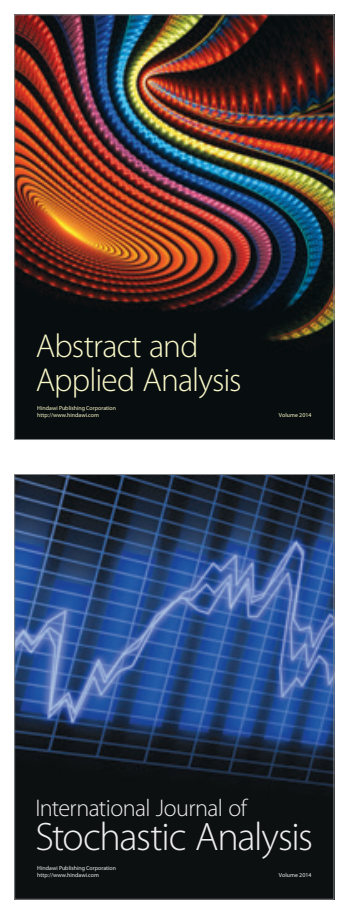

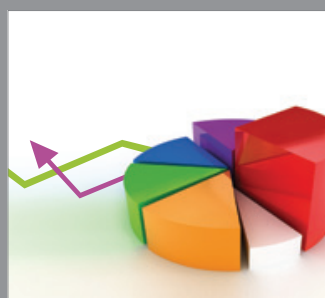

ournal of

Probability and Statistics

Promensencen
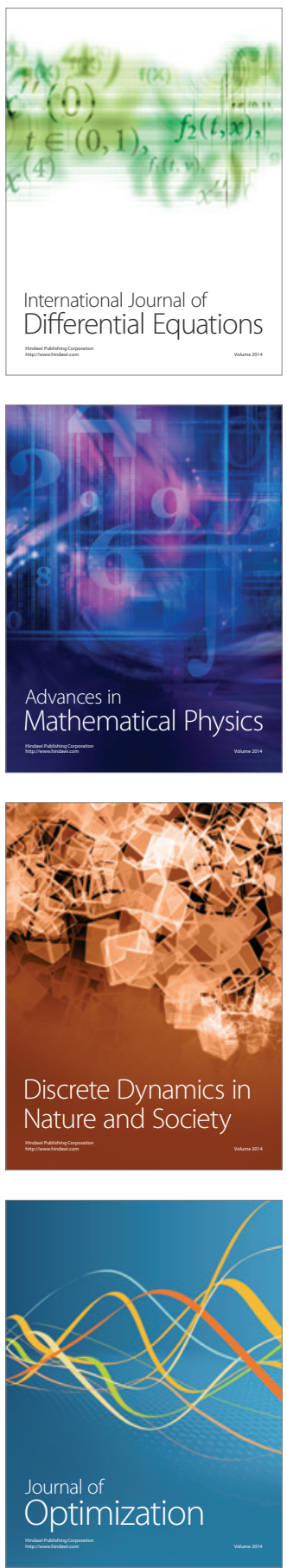